\title{
A CONJECTURE ON COMPACT FRÉCHET SPACES
}

\author{
ZHOU HAO-XUAN
}

\begin{abstract}
Let $X$ be a compact Hausdorff space. $X$ is Frechet if every feebly compact subset is closed in $X$. Under MA, the converse is false.
\end{abstract}

A space $X$ is called Fréchet if $A \subseteq X$ and $x \in \bar{A}$ imply that there is a sequence $\left\{a_{i}\right.$; $i<\omega\}$ in $A$ which converges to $x$. Here and in the sequel, spaces are always assumed to be regular. A space is called feebly compact if every discrete family of open subsets is finite, or equivalently, for every countable open cover there is a finite subcollection such that the closures of its members cover the space. For more characterizations,. see [BCM]. Note that any countably compact space is feebly compact, and feeble compactness is equivalent to countable compactness for normal spaces, or equivalent to pseudocompactness for completely regular spaces.

Recently, Ismail and Nyikos [IN] showed that under (MA or $2^{\omega}<2^{\omega_{1}}$ ), a compact Hausdorff space is sequential if and only if every countably compact subset is closed. Since Fréchet spaces are sequential, a reasonable conjecture is that a compact Hausdorff space is Fréchet if and only if every feebly compact subset is closed. One direction is right, i.e. Y. Tanaka $\left[\mathbf{T}_{2}\right]$ suggested the following.

THEOREM 1. Let $X$ be a countably compact space. If every feebly compact subset of $X$ is closed, then $X$ is Fréchet.

Proof. Suppose $A \subseteq X, x \in \bar{A} \backslash A$ and there are no sequences in $A$ converging to $x$. Obviously, $\bar{A} \backslash\{x\}$ is not closed. If we could show $\bar{A} \backslash\{x\}$ is feebly compact, the contradiction will lead to the conclusion. Indeed, if $\mathscr{Q}$ is a discrete family of nonempty (relatively) open subsets in $\bar{A} \backslash\{x\}, \mathcal{V}=\left\{U \cap A ; U \in Q_{L}\right\}$ is clearly a discrete family of (relatively) open subsets in $A$. Suppose $|\mathscr{Q}| \geqslant \omega$, hence $|\mathfrak{V}|=$ $|\mathscr{Q}| \geqslant \omega$. Take $V_{i} \in \mathcal{V}$ and $x_{i} \in V_{i} \subseteq A(i<\omega)$. Since $\mathscr{U}$ is discrete, $\left\{x_{i} ; i<\omega\right\}$ has no cluster points in $\bar{A} \backslash\{x\}$. Therefore $x$ is the only cluster point of $\left\{x_{i} ; i<\omega\right\}$ in $\bar{A}$, which means $x$ is the limit of $\left\{x_{i} ; i<\omega\right\}$.

A Fréchet space is called fairly Fréchet if $x \in \bar{A} \backslash A$ implies there is a discrete (relative to $A$ ) sequence $\left\{U_{i} ; i<\omega\right\}$ of relatively open subsets $U_{i}$ of $A$ with $x \in \overline{\cup_{i<\omega} U_{i}}$. Note that any hereditarily normal Fréchet space is fairly Fréchet. The next corollary follows directly from the above proof.

COROLlaRY. A countably compact space $X$ is fairly Fréchet if and only if every feebly compact subset is closed.

Received by the editors July 26, 1982 and, in revised form, January 17, 1983.

1980 Mathematics Subject Classification. Primary 54D30, 54D55.

Key words and phrases. Fréchet spaces, feebly compact.

(C)1983 American Mathematical Society $0002-9939 / 83 \$ 1.00+\$ .25$ per page 
Tanaka asked whether the other direction of Theorem 1 is true for countably compact spaces $\left[\mathbf{T}_{\mathbf{1}}\right]$. Theorem 2 shows consistently that it is not, even for compact spaces, i.e. there is a compact Fréchet, nonfairly-Fréchet space. I do not know whether it is absolutely false. In the following, we assume readers to be familiar with the fundamental theory of MA and some of its corollaries, for example, the materials in $[\mathbf{R}]$.

THEOREM 2 (MA). There is a compact, Fréchet, Hausdorff space $X$ with a feebly compact nonclosed subset $Y$.

Proof. Let $C$ be $D^{\omega}$ minus one point where $D=\{0,1\}$.

Let $A_{\alpha}(\alpha<c)$ be all countable subsets of $C$. Let $\Re$ be a countable basis consisting of compact clopen subsets, and $\mathscr{\vartheta}_{\alpha}(\alpha<c)$ be all infinite countable disjoint families of members of $\mathscr{B}$. Now, we are going to inductively define subsets $D_{\alpha}$ of $C$ and new spaces $X_{\alpha}$ with topologies $\tau_{\alpha}$ satisfying:

(a) $D_{\alpha} \subseteq A_{\alpha}$, and if $D_{\alpha}$ is nonempty, then it is an infinite discrete closed subset of $C$

(b) $X_{\alpha}=C \cup\left\{x_{\beta} ; \beta \leqslant \alpha\right\}$, where the $x_{\beta}$ 's may be new points for $\beta>0$;

(c) $\tau_{\alpha}$ is locally compact, zero dimensional, first countable;

(d) for $\alpha_{1}<\alpha_{2}, \tau_{\alpha_{1}} \subseteq \tau_{\alpha_{2}}$;

(e) $\mathcal{Q}_{\alpha}$ is not discrete in $X_{\alpha+1}$;

(f) all $D_{\beta}(\beta<\alpha)$ are still discrete in $X_{\alpha}$ if they are not empty.

If $A_{0}$ is not relatively compact in $C$, i.e. there is an infinite subset without cluster points in $C$, let $D_{0}$ be an arbitrary infinite discrete closed subset of $A_{0}$. Otherwise, let $D_{0}=\varnothing$. Let $X_{0}=C$ and $x_{0} \in C$ be any fixed point. $\tau_{0}$ is the usual topology on $C$.

Suppose $X_{\beta}, x_{\beta}, D_{\beta}, \tau_{\beta}$ have been defined for all $\beta<\alpha$ with properties (a)-(f). Let $X_{\alpha}^{\prime}=\cup_{\beta<\alpha} X_{\beta}$ and $\tau_{\alpha}^{\prime}$ be generated by $\cup_{\beta<\alpha} \tau_{\beta}$. If $\alpha$ is a limit, or $\alpha=\gamma+1$ and $\mathcal{Q}_{\gamma}$ is not discrete in $X_{\alpha}^{\prime}=X_{\gamma}$, define $X_{\alpha}=X_{\alpha}^{\prime}, \tau_{\alpha}=\tau_{\alpha}^{\prime}$ and $x_{\alpha}=x_{0}$.

Now assume $\alpha=\gamma+1$ and $\mathscr{d}_{\gamma}$ is discrete in $X_{\gamma}$. Let $\mathscr{U}_{\gamma}=\left\{B_{i} ; i<\omega\right\}$ and let the $B_{i}$ 's be members of $\mathscr{B}$. Note that since each $B_{i}$ is a CCC compact Hausdorff subspace, and each $D_{\beta}$ is nowhere dense in $C$, so is $D_{\beta} \cap B_{i}$ in $B_{i}$. By Theorem 2 of $[\mathbf{R}], B_{i} \backslash \cup_{\beta<\alpha} D_{\beta} \neq \varnothing$ for all $i<\omega$. Pick $d_{i} \in B_{i} \backslash \cup_{\beta<\alpha} D_{\beta}$. Clearly, $D=\left\{d_{i} ; i<\right.$ $\omega\}$ is an infinite closed discrete subset of $C$.

Consider any nonempty $D_{\beta}$. Let the decreasing family $\left\{G_{i, n} ; n<\omega\right\}$ be a local basis at $d_{i}$ in $C$, where all $G_{i, n} \in \Re$ and $G_{i, n} \subseteq B_{i}$. Since $D_{\beta} \cap D=\varnothing$, there exists a function $f_{\beta}: \omega \rightarrow \omega$ and neighborhoods $G_{i, f_{\beta}(i)}$ of $d_{i}$ for $i<\omega$ such that $D_{\beta} \cap$ $\cup_{i<\omega} G_{i, f_{\beta}(i)}=\varnothing$. By [R, Corollary 11], there is an upper bound $g$ of $\left\{f_{\beta} ; \beta<\alpha\right\}$ such that for each $\beta<\alpha$, there is $n<\omega$ such that $g(k)>f_{\beta}(k)$ for all $k>n$.

Add a new point $x_{\alpha}$ to $X_{\alpha}^{\prime}$ and define $\left\{\left\{x_{\alpha}\right\} \cup \cup_{i>k} G_{i, g(i)} ; k<\omega\right\}$ as its basic neighborhoods. Let $X_{\alpha}=X_{\alpha}^{\prime} \cup\left\{x_{\alpha}\right\}$ and $\tau_{\alpha}$ be defined in an obvious way. It is easy to see that $\tau_{\alpha}$ is locally compact, zero dimensional, first countable and Hausdorff. Moreover, each nonempty $D_{\beta}(\beta<\alpha)$ is still discrete in $X_{\alpha}$ by the above definition of $g$ because $D_{\beta}$ intersects at most finitely many $G_{i, g(i)}$ 's. Besides, (e) is clearly satisfied.

Consider $A_{\alpha}$ for any $\alpha<c$. If $A_{\alpha}$ is relatively compact, i.e. $A_{\alpha}$ is contained in a finite union of compact open subsets in $X_{\alpha}$, let $D_{\alpha}=\varnothing$. In the other case, 
$A_{\alpha} \backslash \cup_{k \leqslant n} U_{k}$ is infinite for any finitely many compact basic open subsets $U_{0}$, $U_{1}, \ldots, U_{n}$ in $X_{\alpha}$. By Corollary 9 of $[\mathbf{R}]$, there is an infinite $D_{\alpha} \subseteq A_{\alpha}$ such that $D_{\alpha} \cap U$ is finite for all compact open subsets $U$ of $X_{\alpha}$; hence $D_{\alpha}$ is a closed discrete subset of $X_{\alpha}$. The induction is completed.

Let $Y=\cup_{\alpha<c} X_{\alpha}$ with a basis $\cup_{\alpha<c} \tau_{\alpha}$ and let $X=Y \cup\{e\}$ be the one-point compactification of $Y$. Finally, we end the proof with two claims.

Claim 1. $X$ is Fréchet.

Obviously, it suffices to check the cases $e \in \bar{A} \backslash A$ for some $A \subseteq X$. If $A \backslash C$ is infinite, since any infinite sequence in $Y \backslash C$ is convergent to $e$ by definition, any infinite sequence in $A \backslash C$ is as desired. Without loss of generality, assume $A \subseteq C$. Since $C$ is hereditarily separable, there is a countable dense subset $A^{\prime}$ of $A$. It follows that $e \in \overline{A^{\prime}} \backslash A^{\prime}$. Suppose $A^{\prime}=A_{\alpha}$ for some $\alpha<c$, then $D_{\alpha}$ is the desired convergent sequence because $A_{\alpha}$ could not be relatively compact in $X_{\alpha}$, and any compact subset of $Y$ only contains finitely many points of $D_{\alpha}$ by (f).

Claim 2. $Y$ is feebly compact.

Let $\mathcal{Q}$ be any infinite disjoint family of open subsets of $Y$. We may assume $Q 1$ is countable and each member of $\mathscr{Q}$ is in $\beta$, or a basic neighborhood of the form $\left\{x_{\beta}\right\} \cup \cup_{i>n} B_{i}$ at a new point $x_{\beta}$. Substitute the basic neighborhood by $B_{i}$ in $\vartheta_{1}$ to get a new family $Q^{\prime}$. It will not affect our discussion if we turn to the consideration of $\mathscr{U}^{\prime}$. Let $\mathscr{U}^{\prime}=\mathcal{Q}_{\alpha}$ for some $\alpha<c$. (e) says $\mathscr{U}_{\alpha}$ is not discrete in $X_{\alpha+1}$, hence in $Y$.

REMARKS. (a) Note that the nonclosed feebly compact subset $Y$ in Theorem 2 has cardinality $c$. By using the Čech-Pospišil technique [K], it is not hard to prove that every feebly compact subset of cardinality less than $c$ is closed in any Fréchet space.

(b) After this paper was accepted, E. van Douwen kindly informed the author that for Theorem 2 Berner [B] had obtained another counterexample under MA. But my proof seems simpler and more direct than his.

\section{REFERENCES}

[B] A. Berner, $\beta(X)$ can he Fréchet, preprint.

[BCM] R. Bagley, E. Connell and J. McKnight, Jr., On properties characterizing pseudo-compact spaces, Proc. Amer. Math. Soc. 9 (1968), 500-506.

[IN] M. Ismail and P. Nyikos, On spaces in which countably compact sets are closed, and hereditary properties, Topology Appl. 11 (1980), 281-292.

[K] K. Kunen, Combinatorics, Handbook of Mathematical Logic (J. Barwise, ed.), North-Holland, Amsterdam, 1977, pp. 371-401.

[R] M. Rudin, Martin's Axiom, Handbook of Mathematical Logic (J. Barwise, ed.), North-Holland, Amsterdam, 1977, pp. 491-501.

[ $\left.T_{1}\right]$ Y. Tanaka, Some results on sequential spaces, Bull. Tokyo (jakugei Univ. (4) 33 (1981), 1-10.

$\left[\mathbf{T}_{2}\right]$ , private communication.

Department of Mathematics, Sichuan University, Chengdu, China 